\title{
Dynamics of a prominence-horn structure during its evaporation in the solar corona
}

\author{
Bing Wang ${ }^{1}$, Yao $\mathrm{Chen}^{1}$, Jie $\mathrm{Fu}^{1,2}$, Bo Li ${ }^{1}$, Xing $\mathrm{Li}^{3}$, and Wei Liu ${ }^{4}$
}

\begin{abstract}
The physical connection among and formation mechanisms of various components of the prominence-horn cavity system remain elusive. Here we present observations of such a system, focusing on a section of the prominence that rises and separates gradually from the main body. This forms a configuration sufficiently simple to yield clues to the above issues. It is characterized by embedding horns, oscillations, and a gradual disappearance of the separated material. The prominence-horn structure exhibits a large amplitude longitudinal oscillation with a period of $\sim 150$ minutes and an amplitude of $\sim 30 \mathrm{Mm}$ along the trajectory defined by the concave horn structure. The horns also experience a simultaneous transverse oscillation with a much smaller amplitude $(\sim 3 \mathrm{Mm})$ and shorter period ( 10-15 minutes), likely representative of a global mode of the large-scale magnetic structure. The gradual disappearance of the structure indicates that the horn, an observational manifestation of the field-aligned transition region separating the cool and dense prominence from the hot and tenuous corona, is formed due to the heating and diluting process of the central prominence mass, while most previous studies suggest that it is the opposite process, i.e., the cooling and condensation of coronal plasmas, to form the horn. This study also demonstrates how the prominence transports magnetic flux to the upper corona, a process essential for the gradual build-up of pre-eruption magnetic energy.
\end{abstract}

Subject headings: Sun: corona — Sun: filaments, prominences — Sun: activity — Sun: coronal mass ejections (CMEs)

\section{Introduction}

Prominences, cool and dense materials in the solar atmosphere, are frequently accompanied by coronal cavities and horn-like structures. This is especially true for high-latitude quiescent

\footnotetext{
${ }^{1}$ Shandong Provincial Key Laboratory of Optical Astronomy and Solar-Terrestrial Environment, and Institute of Space Sciences, Shandong University, Weihai 264209, China; yaochen@sdu.edu.cn

${ }^{2}$ Department of Physics and Astronomy, University of British Columbia, Vancouver, BC V6T 1Z1, Canada

${ }^{3}$ Department of Physics, Aberystwyth University, Aberystwyth, Ceredigion, SY23 3BZ, UK

${ }^{4}$ Stanford-Lockheed Institute for Space Research, Stanford University, Stanford, CA 94305, USA
} 
prominences (e.g. Régnier et al. 2011; Berger et al. 2012; Schmit \& Gibson 2013). Coronal cavities are an elliptical and relatively dark region observed in EUV at typical coronal temperatures (1 $2 \mathrm{MK}$ ). Horns are bright and narrow concave-upward structures emanating from prominences and extending into cavities, best visible in the $171 \AA$ passband of the Atmospheric Imaging Assembly (AIA; Lemen et al. 2012) on board the Solar Dynamic Observatory (SDO; Pesnell et al. 2012). The prominence-cavity system is in general believed to be representative of a flux rope structure consisting of twisted magnetic field lines, which is one major progenitor of solar coronal mass ejections (CMEs; Fan 2001; Fan \& Gibson 2003; Gibson \& Fan 2006; Manchester et al. 2004; Schmit et al. 2009, see Luna et al. (2012) and Schmit et al. (2013) for an alternative scenario with a sheared arcade and overlying unsheared field for the prominence-horn-cavity system). It remains elusive as to how the magnetic energy and flux are gradually transported to and accumulated in the corona, thereby leading to eruptions.

It is generally believed that prominences are suspended at magnetic dips of a larger magnetic field configuration and supported by the magnetic tension force. On the other hand, horns connect the cool and dense prominence to the hot and tenuous corona, representing a field-aligned prominence-corona transition region (e.g., Luna et al. 2012). The morphology of horns indicates that they are field-aligned structures, and can therefore be used to infer the magnetic configuration of the prominence-cavity system. A series of studies suggest that horns are a part of the formation process of prominences from cooling and condensing coronal plasmas (Liu et al. 2012; Berger et al. 2012; Schmit \& Gibson 2013; Schmit et al. 2013; Luna et al. 2012). This has been supported by a detailed examination of spatial and temporal correlations between emission intensities observed in various AIA passbands, for a few sets of horn structures, and supported by radiative magnetohydrodynamic (MHD) simulations (e.g., Luna et al. 2012, Schmit et al. 2013 and Xia et al. 2014). A different scenario of horn formation has been suggested by Fan (2012), who proposed that horns are the current concentration and potential or ongoing reconnection (and thus heated) site around magnetic separatrices of a hyperbolic flux tube. This second scenario is based on the three-dimensional MHD simulations of coronal cavities. However, so far it has no observational support to the best of our knowledge. Here we will present a third scenario to explain the horn formation.

Prominence-filament oscillations have been well observed for nearly a century with ground- and space-based instruments (e.g. Dvson 1930; Newton 1935; Moreton \& Ramsey 1960; Jing et al. 2003; Okamoto et al. 2004; Luna et al. 2014). They can be classified into longitudinal and transverse oscillations according to the orientation of oscillations relative to filament spines (see Shen et al. (2014) for a recent study and more references), and into small (a few $\mathrm{km} \mathrm{s}^{-1}$ ) and large $\left(>10 \mathrm{~km} \mathrm{~s}^{-1}\right.$ ) amplitude oscillations. Small amplitude oscillations are common in the solar atmosphere, whereas large amplitude ones are relatively rare. Prominence oscillations are important because they carry valuable information on the underlying magnetic field structure, which is difficult to measure with present techniques. This line of studies forms a research branch of solar physics, dubbed prominence seismology (e.g. Hyder 1966; Oliver \& Ballester 2002; Tripathi et al. 2009; Arregui et al. 
2012; Luna et al. 2014).

Among various types of prominence oscillations, the most relevant to our study is the largeamplitude longitudinal oscillations (LALOs), first reported by Jing et al. (2003). According to the latest numerical studies (Luna \& Karpen 2012; Luna et al. 2016) the most likely restoring force for LALOs is the field-aligned component of solar gravity, in much the same way that pendulums operate. Indeed, the observed periods seem to be consistent with the pendulum theory. Here in our event not only an LALO itself can be clearly observed, but also we can infer the associated magnetic curvature with the accompanying horn structure. This provides a nice opportunity to test the pendulum theory of LALOs.

Regarding the intimate prominence-horn connection and the rich varieties of prominence oscillations, it is natural to ask whether the horns can also manifest oscillations. The numerical study by Luna et al. (2016) shows that besides the prominence LALO, the large-scale magnetic structure may also support a periodic transverse motion that is interpreted as a global fast normal mode. The horns with their relatively-long field-aligned spatial extension serve as a good magnetic tracer to reveal the existence of such modes, as supported by our novel observation of horn dynamics.

\section{Observational data and event overview}

The prominence examined here is a typical quiescent one, observed by both the AIA/SDO and the $\mathrm{H}_{\alpha}$ images provided by the Global Oscillations Network Group (GONG) project. It appeared in the field of view (FOV) at the northeast limb ( $\sim 5^{\circ}$ in latitude) at 22:00 UT on July 7, 2011. According to Figure 1a-1c and the accompanying animation, the prominence pillar emits at $\mathrm{H}_{\alpha}$ $\left(\sim 10^{4} \mathrm{~K}\right)$ and $304 \AA(\sim 0.08 \mathrm{MK})$, and manifests as a vertical absorption feature at $171 \AA(\sim 0.8$ $\mathrm{MK})$. The southern side of the $\mathrm{H}_{\alpha}$ pillar is connected to the surface by a number of arching threads which are also observable in the hotter 304-171 $\AA$ passbands. Within these threads persistent bidirectional mass flows are observed, indicative of a dynamical equilibrium undergoing significant mass exchange. This is consistent with earlier studies on the mass supply and drainage processes of solar prominences (e.g. Zirker et al. 1998; Berger et al. 2011, 2012; Liu et al. 2012).

From the accompanying animation we see that the upper part of the prominence presents co-incident wavy motion in both the $\mathrm{H}_{\alpha}$ and $304 \AA$ passbands, yet the $\mathrm{H}_{\alpha}$ images have in general poorer quality with less details than the $304 \AA$ data. In addition, some structures appear above the prominence pillar at $304 \AA$ while invisible at $\mathrm{H}_{\alpha}$. This is likely due to the progressively more rarefied and hotter condition of the emitting materials associated with the rising flux tube higher in the corona. Dynamics of these structures observed by AIA at 304 and $171 \AA$ are the focus of our study.

It can be seen that the prominence has a similar morphology in the $171 \AA$ emission (Figure $1 \mathrm{~b}$ ), on top of which we observe a group of concave-upward structures that have been dubbed prominence "horns" (e.g. Berger et al. 2012) or "U-shape structures" (Régnier et al. 2011). Consistent with 
previous studies, the horns emanate from the corresponding prominence and extend upward into the corona. In Figure 1d, we present the AIA $211 \AA$ image recorded at 03:28 UT on July 10, which shows the associated cavity on top of the prominence-horn structure. Although the cavity is only clearly visible after 02:00 UT on July 10, the overall prominence-horn morphology does not change much. We therefore suggest that the underlying structure, presumably a flux rope, has been present during the earlier period of interest.

The prominence-cavity structure starts to rise gradually at 08:00 UT and erupts at 11:30 UT on July 10, leading to a CME propagating at a linear speed of $\sim 427 \mathrm{~km} \mathrm{~s}^{-1}$ according to the Large Angle Spectroscopic Coronagraph (LASCO; Brueckner et al. 1995) C2 data. The pre-eruption prominence is observed on the disk by the Solar TErrestrial RElations Observatory (STEREO; Kaiser et al. 2008) B, yet it is very faint, possibly due to its high altitude as indicated by the simultaneous AIA images and its relatively weak emission in comparison with other disk features. The eruption results in a large-scale arcade structure in the corona according to the STEREO-B $304 \AA$ data (not shown here), with flare-like ribbons sweeping across a region $\sim 50^{\circ}$ long in longitude and $\sim 400 \mathrm{Mm}$ (55 arcsecs) wide. This suggests that the pre-eruption prominence-cavity system is a well-developed, hemispheric-scale magnetic structure.

Here we focus our study on the prominence-horn dynamics observed one day before the eruption. Of particular interest is the dynamical evolution when a small upper section of the prominence rises and separates from the underlying main prominence structure. At 19:00 UT on July 8, this section, together with the embedded horns, appears mostly isolated from the main structure (See the region S1 in Figure 1e-1f and the animation). We speculate that the separation and the accompanying large scale motion are possibly due to reconnections, prominence structural changes or a mass drainage (or unloading) process that destroys the original force balance around the top of the prominence.

This upper section then ascends gradually into the overlying structure which has been suggested to be a flux rope and manifested later as a corona cavity (see Figure 1d). The suspending prominence-horn structure exhibits a large-amplitude oscillation while slowly fading from the FOV and disappearing completely around 20:50 UT. We concentrate on the prominence-horn dynamics from the upper prominence section starts to ascend ( 17:00 UT) to its disappearance. While the structure of interest is also visible at $131 \AA$ through its low-temperature response $(\sim 0.5 \mathrm{MK})$, the emission is too weak to reveal useful information.

Because the main prominence body is structurally complex and highly dynamic, only the significant separation of the small upper section from the main body as well as its projection onto the relatively dark coronal background allows us to clearly isolate this portion of the prominence for further analysis. 


\section{Main results}

As is evident from the animation accompanying Figure 1, the upper part of the prominence exhibits a large-scale motion along a curved trajectory that basically follows the curvature of the embedding horns.

The motion can be seen from Figure 2 which presents the distance-time map of S1, in which the $304 \AA$ intensities have been averaged along the vertical direction. We can see that the prominence starts to move northwestward at $\sim 16: 30 \mathrm{UT}$, and reaches the end at $\sim 17: 10 \mathrm{UT}$, after traversing a distance of $\sim 15 \mathrm{Mm}$. Then the prominence reverses its direction and moves southeastward before moving northwestward again. During this process, the prominence disintegrates into several pieces with each piece accompanied by thread-like horn structures. The bulk collective motion cannot be clearly defined after 19:00 UT at $304 \AA$ and 19:30 UT at $171 \AA$. As mentioned, this large-scale prominence motion is basically along the associated field-aligned horn features, and therefore in accordance with the observational manifestation of LALOs, albeit the motion does not even finish a complete cycle. The period of our LALO is estimated to be $\sim 150$ minutes and the amplitude is $\sim 30 \mathrm{Mm}$, consistent with earlier LALO reports (e.g. Jing et al. 2003, 2006; Vršnak et al. 2007; Shen et al. 2014; Luna et al. 2014).

The restoring force of the LALO has been suggested to be the field-aligned component of the gravity, similar to a pendulum with a length given by the curvature radius of the supporting magnetic structure. The presence of accompanying horns allows us to measure the associated magnetic curvature radius. This provides a nice opportunity to test the pendulum theory of LALOs. To do this, we select several clear horn structures during the LALO and measure their curvature radii. The selected horns are plotted with dashed curves in Figure 3, and are fitted with circular arcs. The curvature radii thus found are 73, 70, and $66 \mathrm{Mm}$ for the three horns, respectively. The corresponding average is $\sim 70 \mathrm{Mm}$, leading to an expected pendulum period of $\sim 1 \mathrm{~h}$. This is considerably less than the measured value of $\sim 2.5 \mathrm{~h}$. Possible factors leading to this discrepancy will be discussed in our final section.

To explore the horn dynamics during the above process, in Figure 4 we plot distance-time maps along three slices across the horns (S2 - S4, see Figure 3). We see that the eastern part of the horn H1 manifests a clear quasi-periodic oscillation (See also the online animation). The oscillation is mostly perpendicular to the horn, which is suggested to delineate a bundle of magnetic field lines, and thus representative of a transverse mode. It is clearly observed from 17:00 - 18:00 UT, lasting for several periods. The period is $\sim 10-15$ minutes and the amplitude is $\sim 3 \mathrm{Mm}$. Both parameters, much smaller than those of the LALO, are comparable to earlier results of smallamplitude transverse prominence oscillations (SATPOs). Since horns emanate from the prominence, it is natural to surmise that the horn oscillations are associated with the SATPO. From the S3 map, which crosses the middle part of the prominence, there seems to exist a weak oscillation with a smaller amplitude and a similar period, yet the feature is diffuse and our statement is not conclusive. An inspection of the accompanying animation shows that the western part of two nearby horns 
also presents some oscillations with seemingly-opposite phases. However, the features of these oscillations can be hardly recognized from the distance-time map along S4 (Figure 4c), due to the rather weak emissivity there. In other intervals, e.g., after 19:30 UT, signatures of horn oscillations with comparable periods can be seen from the first two panels of Figure 4, indicating that the horn motion investigated here possibly represents a persistent global transverse mode supported by the large-scale magnetic structure.

Now we examine the emission features that might reveal some clues to the formation mechanism of horns. We first plot the 304 and $171 \AA$ emission intensity variation along the 3 horns (see Figure 3 ) in Figure 5a-5c. The intensities have been averaged over 5 pixels transversely. We see that the $171 \AA$ intensity tends to reach a high level around the $304 \AA$ intensity maximum. Yet, the details differ for different horns. For H1, the $171 \AA$ emission reaches a local minimum, anti-correlated with the $304 \AA$ intensity profile, while for H2 and H3 the $171 \AA$ emissions reach a plateau of their intensities around the $304 \AA$ maxima. These observations are not inconsistent with those modelled by Luna et al. (2012) with a simplified one-dimensional thermal non-equilibrium model of coronal condensation and prominence formation. They showed that the horns at $171 \AA$ are characterized by brightenings on both sides of a central $304 \AA$-emitting mass. From the central part towards ends along the horns, both 171 and $304 \AA$ emissions decline gradually. Note that the 171 intensity always declines slower and occupies a broader emitting region than its 304 counterpart.

Overall, the 171 and $304 \AA$ emissions are strongly correlated, as clearly seen from their correlated variation trends shown in the four panels of Figure 5. The last panel (5d) presents their intensity curves, given by the corresponding total normalized intensities in S1. We see that the two curves reach the maximum intensity at 18:47 (304 $\AA$ ) and 18:57 (171 $\AA$ ) UT, respectively, before gradually fading out. It seems that the normalized 171 emission maximum is somehow delayed by $\sim 10$ minutes. A similar delay is also indicated by the later knee-like feature around 19:10-19:20 UT. The significance of this delay shall be further investigated and testified with more events.

The observed close correlation of the 171 and 304 emission intensities has been demonstrated previously by Schmit et al. (2013) who suggested that horns are formed due to the cooling-condensation process of coronal plasmas, associated with the prominence formation. In our case the dynamical and morphological evolution of horns is observed during the gradual disappearance of a prominence section. This strongly indicates that the opposite process, referred to as the prominence evaporation, might take place here with the horns being formed by the heating and diluting process of prominence plasmas.

As clearly seen from the animation accompanying Figure 1, the ascending part of the prominence gradually disappears into the upper coronal magnetic structure that is observed one-day later as a cavity (presumable a flux rope structure, see Figure 1d). During this process, the structure disintegrates into several parts which are initially tied together through the prominence mass. Each part carries a horn structure that is representative of a bundle of field lines. Thus, we suggest that the observed prominence ascent and evaporation actually represent a process of the magnetic flux 
(as well as mass and magnetic energy) transport from the large-scale prominence to the upper cavity structure. It is observable here because of the presence of the field-tracing horns. This process certainly contributes to the gradual flux accumulation and energy build-up in the pre-eruption corona, and may play a role in the onset of the one-day-later CME.

\section{Summary and discussion.}

Various dynamical processes of a prominence-horn structure are investigated to provide insights into the prominence-horn cavity connection and accompanying oscillations. The event is observed during a gradual rising and a likely-evaporation process of a prominence section that separates from the main body. The prominence section of interest, with its embedded horns, experiences a large amplitude oscillation with a period of $\sim 2.5 \mathrm{~h}$ and an amplitude of $\sim 30 \mathrm{Mm}$ along the trajectory defined by the concave-outward horn structure. The simultaneous observation of this LALO and the associated horn structure offers a nice opportunity to test the pendulum theory of the LALO and it is found that the predicted period is significantly shorter than observed.

The mechanisms underlying the possible evaporation could not be determined with available data. Presumptions include the field-aligned thermal conduction from the hot corona plasmas towards the cool prominence plasmas, changes of the amount and spatial distribution of heating along the flux tube with horns (see, e.g., Luna et al., 2012), and/or waves- or turbulence- related heating processes within or outside the cool prominence section. In addition, the rising of the prominence-horn structure into the higher corona may cause the field line to expand with a shallower dip geometry, and carry the cool dense material into a less dense environment. This may also contribute to the draining and evaporation of the prominence material.

As mentioned in the introduction, latest numerical studies (Luna et al. 2012, 2016) suggest that the LALO is mainly controlled by the gravitational force, and its damping is directly related to the rate of mass accretion that is induced by footpoint-heating-caused chromospheric evaporation and further condensation. Our LALO event takes place during the gradual disappearance of cool materials. Therefore, the mass accretion is not important here. We propose several factors that may contribute to the above-mentioned discrepancy. First of all, since in our case the prominence moves higher to a progressively more rarefied environment with emitting materials contained by a rising flux tube of weaker magnetic field, the relative importance of the gravity and the thermal coupling process in governing the plasma dynamics may differ from their roles in the static flux tubes investigated numerically. This, demanding a further study, may partially account for the reported discrepancy.

Other possible factors include (1) the projection effect of the horn structure that leads to an underestimate of the curvature radius and thus the expected pendulum period, (2) the overall deviation of the magnetic structure away from the radial direction thus making only part of the solar gravity the restoring force, also resulting in an underestimate of the predicted period, and (3) 
the thermal and magnetic coupling between the rising part and the lower main body may enhance the restoring force and thus reduces the period. Among these factors, the effect of the second one can be roughly estimated here. From our data, the horn structure, along which the LALO takes place, is at an angle of $\leq 20$ degrees away from the radial direction, resulting in a slightly longer period $(\sim 1.1 \mathrm{~h})$ than predicted using the total gravity, yet still much shorter than observed $(\sim 2.5 \mathrm{~h})$.

In addition to the LALO, the horns also undergo a transverse oscillation with a smaller amplitude $(\sim 3 \mathrm{Mm})$ and shorter period ( 10-15 minutes). This oscillation seems to be carried by several horns across an extended region and lasts for a relatively long time. This finding is consistent with the latest numerical study of prominence oscillations by Luna et al. (2012, 2016), which reveals the persistent existence of a global fast collective mode of the magnetic structure that supports an oscillating prominence. Their results are comparable to those reported here for the small-amplitude transverse horn oscillations, indicating that the oscillation is likely the fast collective mode supported by the overall magnetic structure. More studies are required to further explore the seismological potential of this kind of oscillations.

As mentioned in Section 1, currently available scenarios to explain the horn formation in-

clude coronal condensation (Berger et al. 2012; Schmit \& Gibson 2013; Schmit et al. 2013), and the current-sheet concentration surface of a hyperbolic flux tube (Fan 2012). What we report here shows that the horn structure emanates from the prominence and gradually fades away. This strongly indicates that the horn formation may also be attributed to the prominence evaporation, likely involving some heating and diluting process of the central prominence mass. This can be regarded as a scenario opposite to the present cooling-condensation scenario of horn formation. In the corona, it may go both ways, heating or cooling, both with a transition between cool and hot mass.

Using the horn structure as tracers of the underlying magnetic structure, our study also illustrates how the prominence can transport magnetic flux to the overlying cavity-flux rope structure, and thus to increase the magnetic energy there. This may be a fundamental process essential for the gradual build-up of magnetic energy in the pre-eruption corona, especially in the cavity region overlying a prominence structure.

We gratefully acknowledge the usage of data from the SDO, STEREO, SOHO spacecraft and the ground-based GONG project. This work was supported by grants NNSFC 41331068, 41274175 (YC), 41274176, 41474149 (BL), and NSBRSF 2012CB825601.

\section{REFERENCES}

Arregui, I., Oliver, R., \& Ballester, J. L. 2012, Living Reviews in Solar Physics, 9, doi:10.12942/lrsp$2012-2$ 
Berger, T. 2014, in IAU Symposium, Vol. 300, Nature of Prominences and their Role in Space Weather, ed. B. Schmieder, J.-M. Malherbe, \& S. T. Wu, 15-29

Berger, T., Testa, P., Hillier, A., et al. 2011, Nature, 472, 197

Berger, T. E., Liu, W., \& Low, B. C. 2012, ApJ, 758, L37

Brueckner, G. E., Howard, R. A., Koomen, M. J., et al. 1995, Sol. Phys., 162, 357

Dyson, F. 1930, MNRAS, 91, 239

Fan, Y. 2001, ApJ, 554, L111

Fan, Y. 2012, ApJ, 758, 60

Fan, Y., \& Gibson, S. E. 2003, ApJ, 589, L105

Gibson, S. E., \& Fan, Y. 2006, Journal of Geophysical Research (Space Physics), 111, A12103

Hyder, C. L. 1966, ZAp, 63, 78

Jing, J., Lee, J., Spirock, T. J., et al. 2003, ApJ, 584, L103

Jing, J., Song, H., Abramenko, V., Tan, C., \& Wang, H. 2006, ApJ, 644, 1273

Kaiser, M. L., Kucera, T. A., Davila, J. M., et al. 2008, Space Sci. Rev., 136, 5

Lemen, J. R., Title, A. M., Akin, D. J., et al. 2012, Sol. Phys., 275, 17

Liu, W., Berger, T. E., \& Low, B. C. 2012, ApJ, 745, L21

Luna, M., \& Karpen, J. 2012, ApJ, 750, L1

Luna, M., Karpen, J. T., \& DeVore, C. R. 2012, ApJ, 746, 30

Luna, M., Knizhnik, K., Muglach, K., et al. 2014, ApJ, 785, 79

Luna, M., Terradas, J., Khomenko, E., Collados, M., \& de Vicente, A. 2016, ApJ, 817, 157

Manchester, IV, W., Gombosi, T., DeZeeuw, D., \& Fan, Y. 2004, ApJ, 610, 588

Moreton, G. E., \& Ramsey, H. E. 1960, PASP, 72, 357

Newton, H. W. 1935, MNRAS, 95, 650

Okamoto, T. J., Nakai, H., Keiyama, A., et al. 2004, ApJ, 608, 1124

Oliver, R., \& Ballester, J. L. 2002, Sol. Phys., 206, 45

Pesnell, W. D., Thompson, B. J., \& Chamberlin, P. C. 2012, Sol. Phys., 275, 3 
Régnier, S., Walsh, R. W., \& Alexander, C. E. 2011, A\&A, 533, L1

Schmit, D. J., \& Gibson, S. 2013, ApJ, 770, 35

Schmit, D. J., Gibson, S., Luna, M., Karpen, J., \& Innes, D. 2013, ApJ, 779, 156

Schmit, D. J., Gibson, S. E., Tomczyk, S., et al. 2009, ApJ, 700, L96

Shen, Y., Liu, Y. D., Chen, P. F., \& Ichimoto, K. 2014, ApJ, 795, 130

Tripathi, D., Isobe, H., \& Jain, R. 2009, Space Sci. Rev., 149, 283

Vršnak, B., Veronig, A. M., Thalmann, J. K., \& Žic, T. 2007, A\&A, 471, 295

Xia, C., Keppens, R., Antolin, P., \& Porth, O. 2014, ApJ, 792, L38

Zirker, J. B., Engvold, O., \& Martin, S. F. 1998, Nature, 396, 400 

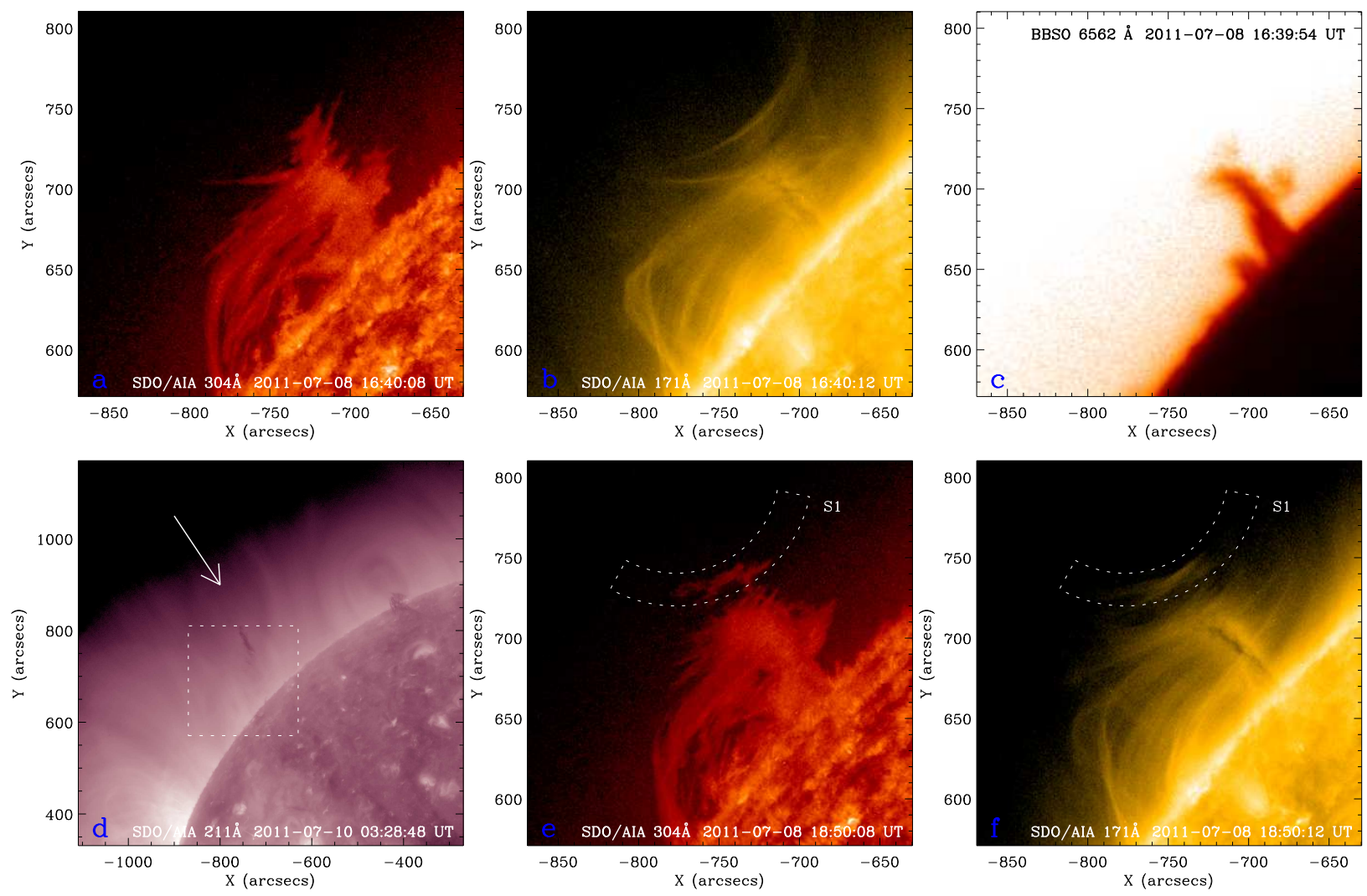

Fig. 1.- Overview of the prominence-horn cavity system and the associated eruptive features. (a-c) The asymmetric morphology of the large-scale prominence structure observed in the 304-171 $\AA$ and $\mathrm{H}_{\alpha}$ emissions. (d) The cavity structure at $211 \AA$, pointed at by a white arrow. The box indicates the FOV of other panels. (e-f) The separation of the oscillating upper part. The curved slice (S1) is for the distance-time map shown in Figure 2, with a starting point located at the "S1" end. An animation of this figure is available (with negative images for better visual effect). 


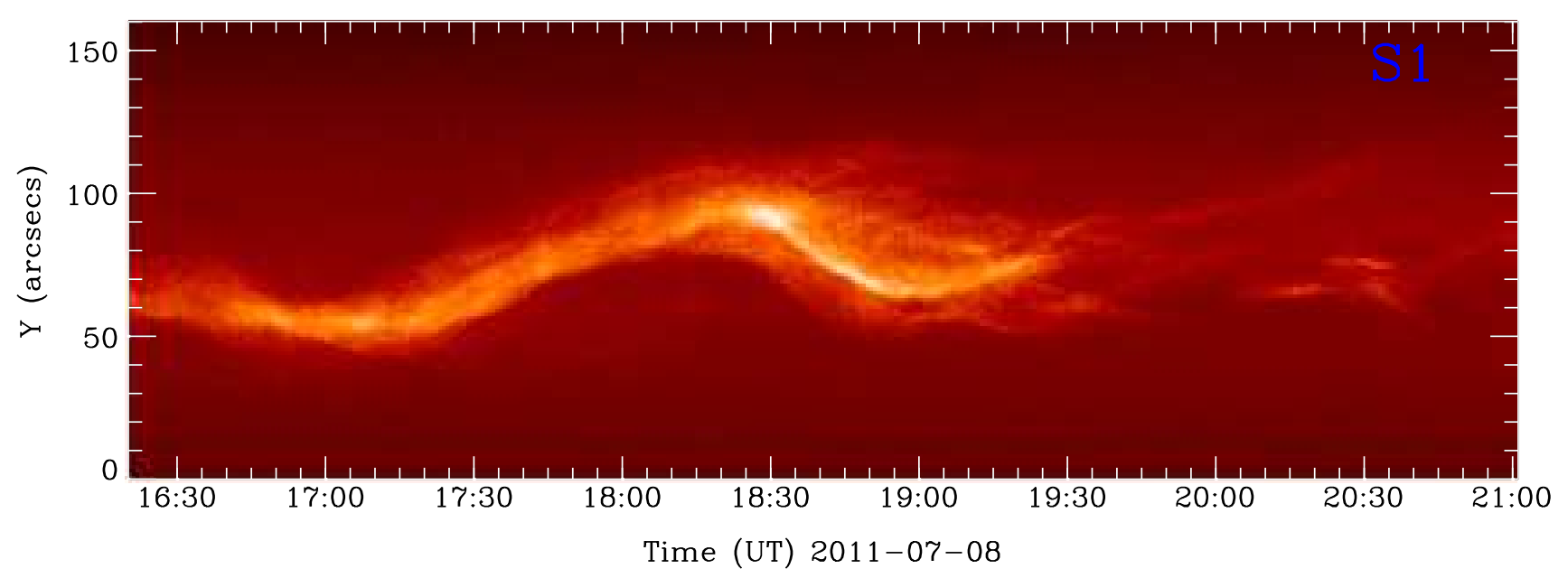

Fig. 2.- Distance-time maps at $304 \AA$ along the slice S1 presented in Figure 1e-1f, to show the prominence LALO motion. The slice is 268 pixels long and 35 pixels wide. 

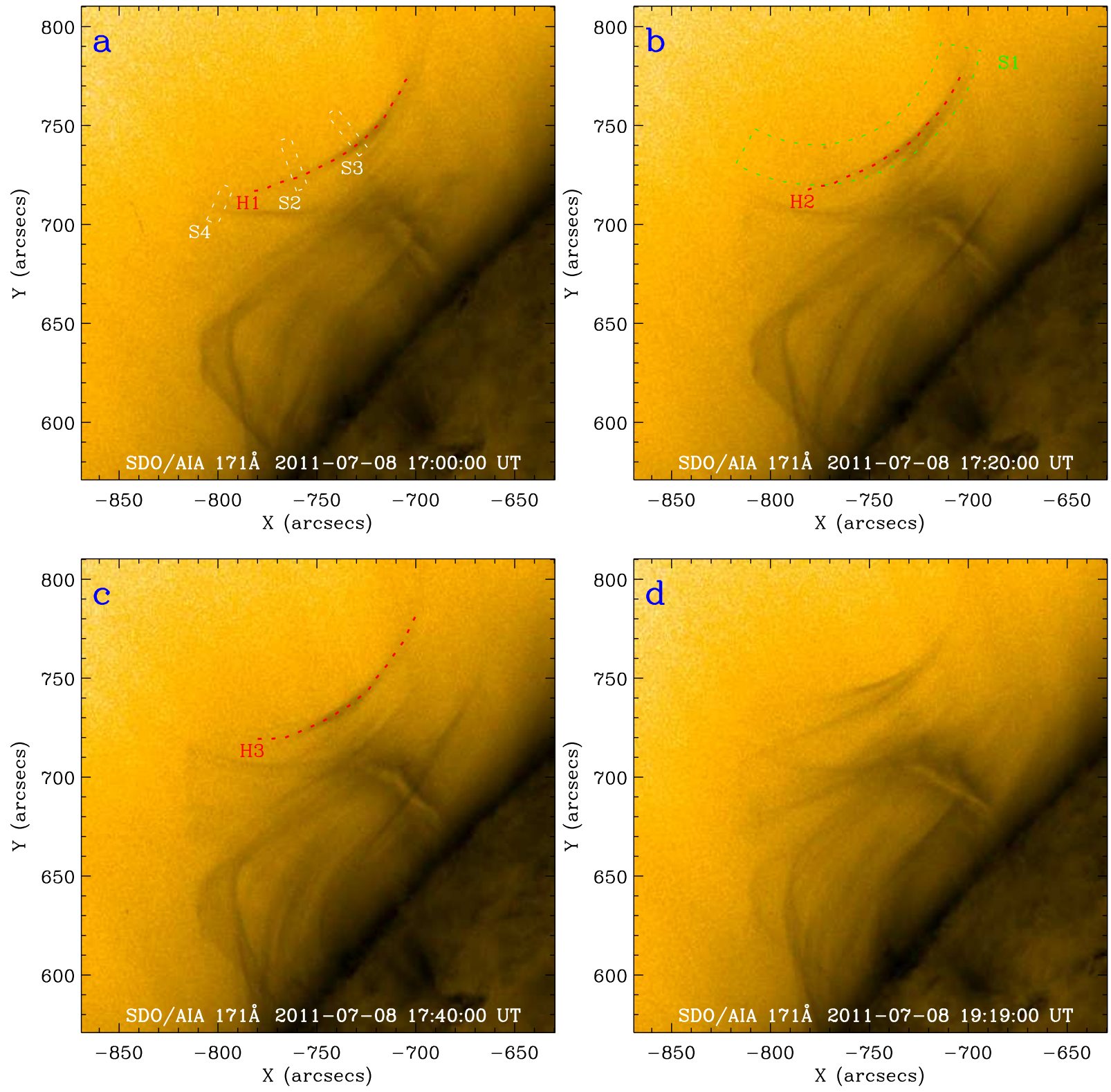

Fig. 3.- Selected horns at different LALO phases in negative images. The horn profiles are fitted with red dashed circular arcs. S2-S4 are slices to obtain the distance-time maps across horns (Figure 4), H1-H3 are to plot the horn flux curves (Figure 5). Their starting points are at the end close to the labels. 


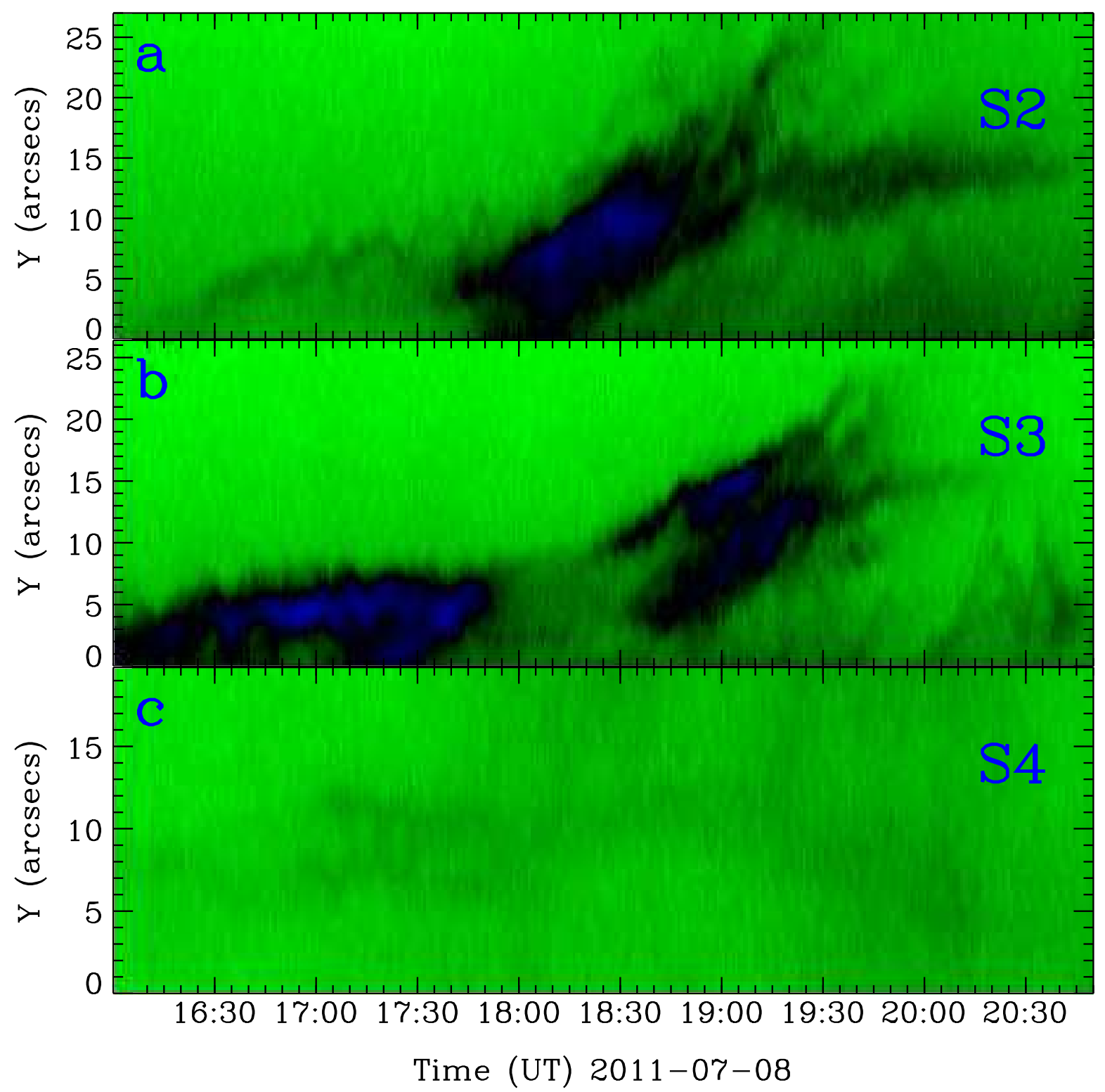

Fig. 4.- Distance-time maps at $171 \AA$ along slices (S2 - S4), to show the quasi-periodic transverse oscillations of the horns. An animation of this figure is available. 

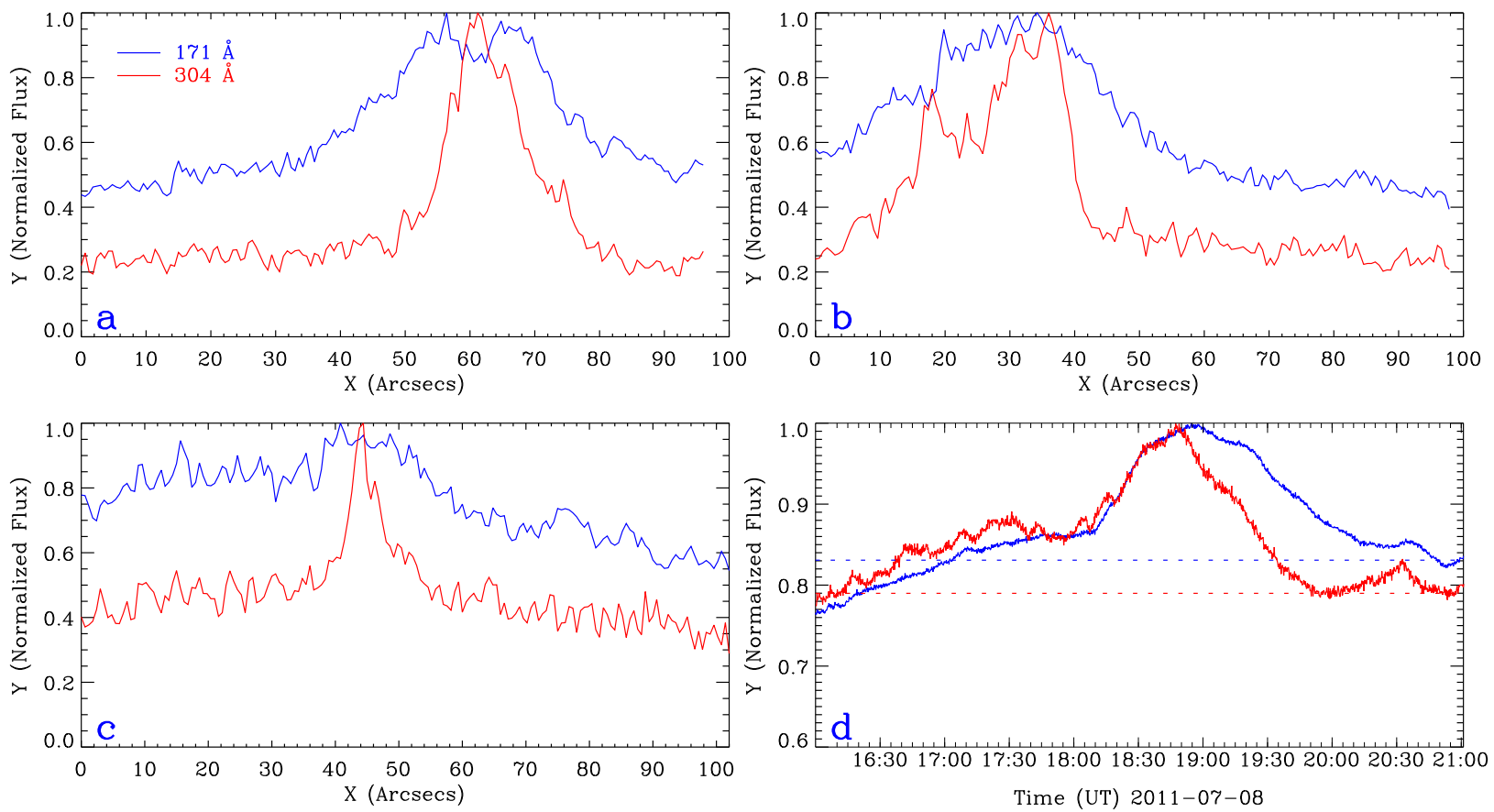

Fig. 5.- Correlation of the AIA 304 (red) and 171 (blue) $\AA$ intensities. (a - c) Normalized intensity variations along the horns (H1 - H3). (d) The 304 and $171 \AA$ emission intensities integrated over the window S1, with the background levels represented by the horizontal dashed lines. 